\title{
REQUEST - A RECURSIVE QUEST ALGORITHM \\ FOR SEQUENTIAL ATTITUDE DETERMINATION
}

\author{
Itzhack Y. Bar-Itzhack \\ Flight Dynamics Support Branch, Code 553 \\ NASA Goddard Space Flight Center \\ Greenbelt, MD 20771
}

\begin{abstract}
In order to find the attitude of a spacecraft with respect to a reference coordinate system, vector measurements are taken. The vectors are pairs of measurements of the same generalized vector, taken in the spacecraft body coordinates, as well as in the reference coordinate system. We are interested in finding the best estimate of the transformation between these coordinate systems. The algorithm called QUEST yields that estimate where attitude is expressed by a quatemion. QUEST is an efficient algorithm which provides a least squares fit of the quatemion of rotation to the vector measurements. QUEST, however, is a single time point (single frame) batch algorithm, thus measurements that were taken at previous time points are discarded.
\end{abstract}

The algorithm presented in this work provides a recursive routine which considers all past measurements. The algorithm is based on the fact that the, so called, $K$ matrix, one of whose eigenvectors is the sought quaternion, is linearly related to the measured pairs, and on the ability to propagate $\mathrm{K}$. The extraction of the appropriate eigenvector is done according to the classical QUEST algorithm. This stage, however, can be eliminated, and the computation simplified, if a standard eigenvalue-eigenvector solver algorithm is used. The development of the recursive algorithm is presented and illustrated via a numerical example.

\section{INTRODUCTION}

The problem of finding attitude from vector observations is stated as follows. A sequence, $b_{i}, i=1,2$, $\ldots, k$ of unit vectors is given. These unit vectors are the result of measurements performed in vehicle cartesian coordinates, of the directions to known objects. The sequence, $r_{i}, i=1,2, \ldots, k$ of unit vectors, is the sequence of the corresponding unit vectors, resolved in a reference cartesian coordinate system. We wish to find the attitude matrix, A, which transforms vectors from the reference to the body coordinates. Obviously, $A$ has to be an orthogonal matrix. In 1965, Wahba posed this problem as a least squares problem as follows. Let

$$
L(A)=\frac{1}{2} \sum_{i=1}^{k}\left|b_{i}-A r_{i}\right|^{2}
$$

find that orthogonal $3 \times 3$ matrix, A, that minimizes $L$. We can weigh each measurement separately according to the accuracy of the particular vector measurement. In addition, we may want to find the quaternion, rather than the matrix, representation of attitude. In such case (1) is replaced by

\footnotetext{
-Sophie and William Shamban Professor of Aerarpace Engineering. On cabbatical leave from the Faculty of Aerospace Engineering. Technion-Irrael Inctinute of Technology, Haifa 32000, Itrnel. Member Technion Space Research Inatiute. IEEE Fellow. ALAA Associate Fellow.
}

This work was performed on a National Research Council NASA Research Aseociateship. 


$$
J(q)=\frac{1}{2} \sum_{i=1}^{k} a_{i}\left|b_{i}-A(q) r_{i}\right|^{2}
$$

where $a_{i}, i=1,2, \ldots, k$ are the positive weights assigned to each measurement. In (2) we are looking for that quaternion, $q$, which minimizes $J$. Note that instead of minimizing $J$, we can maximize $g$ defined as

$$
g(q)=1-J(q)
$$

It can be shown ${ }^{2,3}$ that $g(q)$ can be written as

$$
g(q)=q^{T} K q
$$

where $\mathrm{K}$ is constructed as follows. Define

$$
\begin{gathered}
m_{k}=\sum_{i=1}^{k} a_{i} \\
\sigma=\frac{1}{m_{k}} \sum_{i=1}^{k} a_{i} b_{i}^{T} r_{i} \\
B=\frac{1}{m_{k}} \sum_{i=1}^{k} a_{i} b_{i} r_{i}^{T} \\
S=B+B \\
z=\frac{1}{m_{k}} \sum_{i=1}^{k} a_{i}\left(b_{i} \times r_{i}\right)
\end{gathered}
$$

Then

$$
\mathrm{K}=\left[\begin{array}{c|c}
\mathrm{S}-\sigma \mathrm{I} & \mathrm{z} \\
\hline \mathrm{z}^{\mathrm{T}} & \bar{\sigma}
\end{array}\right]
$$

where $I$ is the third order identity matrix. It was shown ${ }^{2,3}$ that $q^{*}$, of unity length, which maximizes $g(q)$ in (4), satisfies the equation

$$
\mathrm{K} \mathbf{q}^{*}=\lambda \mathbf{q}^{*}
$$

where $\lambda$ is $a$, yet undetermined, Lagrange multiplier. We realize that $\lambda$ is an eigenvalue of $K$ and $q^{*}$ is the eigenvector which corresponds to $\lambda$. Substitution of this solution into (4) yields

$$
g\left(q^{*}\right)=\lambda
$$

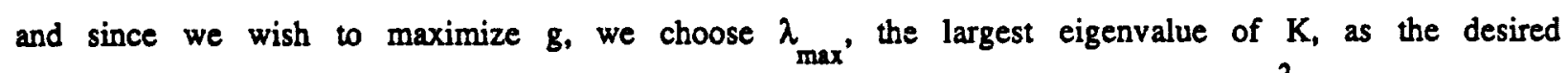
eigenvalue, and then, $q^{*}$ is the eigenvector which corresponds to this $\lambda_{\max }$. Davenport ${ }^{2}$ showed that once $\lambda_{\max }$ is found, there is no need to solve for the eigenvector of $K$, since $y^{*}$, the optimal vector of Rodrigues parameters ${ }^{4}$, (also known as Gibbs vector ${ }^{5}$ ) can be computed as follows 


$$
y^{*}=\left[\left(\lambda_{\max }+\sigma\right) I-S\right]^{-1} z
$$

and the optimal quaternion can be found using the known relation

$$
\mathbf{q}^{*}=\frac{1}{\sqrt{1+\left|\mathbf{y}^{*}\right|^{2}}}\left[\begin{array}{l}
\mathbf{y}^{*} \\
1
\end{array}\right]
$$

Shuster ${ }^{6,7}$ showed how to, easily, compute $\lambda_{\max }$ to arbitrary accuracy, and how to deal with a singular matrix in (9). It was also shown there that $\lambda_{\max }$ is close to 1 and is exactly 1 when the measurements are error-free. (This property is due to the fact that all $a_{i}$ 's in (2) add up to 1 , or, equivalently, the introduction of the normalizing factor $m_{k}$ in to $\left(5 . b, c\right.$, and e)). The algorithm for obtaining $\lambda_{\max }$ and $\mathbf{q}^{*}$ from vector observations discussed above is known as the QUEST algorithm.

QUEST is a single point attitude determination algorithm; that is, it utilizes the vector measurements obtained at a single time point and uses them, and them only, to determine the attitude at that time point. This way, the information contained in past measurements, is lost. This fact has been recognized and in 1989, Shuster presented an algorithm which he named Filter QUEST that processes vector measurements recursively. The Attitude Profile Matrix, B, defined in (5.c), which plays a central role in the algorithm, is updated recursively for use in the QUEST algorithm. Much attention is given, in that paper, to covariance calculations.

In the present work, the matrix, which is updated recursively, is the $K$ matrix defined in (5) and (6). Indeed, as can be seen in the algorithm described above, $\mathrm{K}$ is the most important element in QUEST. In the following section, we start our presentation of REQUEST by considering, first, the recursive time-invariant algorithm. Then, in Section III, we develop the recursive algorithm for the time-varying case and present an example. In Section IV we list the algorithm in a unified form. Finally, in Section $\mathrm{V}$, we present our conclusions and recommendation for further work.

\section{THE RECURSIVE TIME-INVARIANT ALGORITHM}

Assume that the body axes are non-rotating with respect to the reference axes. Also assume that $k$ vectors have been processed using the QUEST algorithm.

Let

$$
m_{k}=\sum_{i=1}^{k} a_{i}
$$

where $m_{k}$ is not necessarily equal to 1 . Also define

$$
\begin{gathered}
\sigma_{k}=\frac{1}{m_{k}} \sum_{i=1}^{k} a_{i} b_{i}^{T} r_{i} \\
B_{k}=\frac{1}{m_{k}} \sum_{i=1}^{k} a_{i} b_{i} r_{i}^{T} \\
S_{k}=B_{k}+B_{k}^{T}
\end{gathered}
$$




$$
z_{k}=\frac{1}{m_{k}} \sum_{i=1}^{k} a_{i}\left(b_{i} \times r_{j}\right)
$$

The parameters $\sigma_{k}, S_{k}$ and $z_{k}$, are then used to compute $K$, which for the case of $k$ measurements, is denoted by $\mathrm{K}_{\mathrm{k}}$. The latter is used to find the optimal quatemion, based on $k$ pairs of measured vectors. (Note that QUEST doesn't require the computation of $K_{k}$ itself). As mentioned earlier, the coefficient $\mathrm{m}_{k}$ is used in (11.b, $c$, and e) to normalize the weights, $a_{i}$, such that $\lambda_{\max }$ is closed to 1 . (See Ref. 5 for the solution of $\lambda_{\max }$ ). Now suppose that an additional measurement has been acquired; that is, the $k+1$ st pair has to be processed. The question is then, do we have to re-compute the $\mathrm{K}_{k+1}$ matrix anew or can we, perhaps, update $K_{k}$ to included the added pair. As will be shown next, the latter is possible. In fact, it forms the basis for the REQUEST algorithm. We formulate this quality of $K$ in the following proposition.

Proposition 1: Let

$$
\begin{gathered}
\delta \sigma_{k+1}=a_{k+1} b_{k+1}^{T} r_{k+1} \\
\delta B_{k+1}=a_{k+1} b_{k+1} r_{k+1}^{T} \\
\delta S_{k+1}=\delta B_{k+1}+\delta B_{k+1}^{T} \\
\delta z_{k+1}=a_{k+1}\left(b_{k+1} \times r_{k+1}\right) \\
=\left[\frac{\delta S_{k+1}-\delta \sigma_{k+1} I}{\delta z_{k+1}^{T}} \mid \frac{\delta z_{k+1}}{\delta \sigma_{k+1}}\right]
\end{gathered}
$$

then

$$
K_{k+1}=\frac{m_{k}}{m_{k+1}} K_{k}+\frac{1}{m_{k+1}} \delta K_{k+1}
$$

Proof: By definition

Now, it can be easily verifies that

$$
\mathrm{m}_{\mathrm{k}+1}=\sum_{\mathrm{i}=1}^{\mathrm{k}+1} \mathrm{a}_{\mathrm{i}}
$$

$$
\begin{aligned}
& \sigma_{k+1} \triangleq \frac{1}{m_{k+1}} \sum_{i=1}^{k+1} a_{j} b_{i}^{T} r_{i}=\frac{1}{m_{k+1}} \sum_{i=1}^{k} a_{i} b_{i}^{T} r_{j}+\frac{1}{m_{k+1}} a_{k+1} b_{k+1}^{T} r_{k+1} \\
& B_{k+1} \triangleq \frac{1}{m_{k+1}} \sum_{i=1}^{k+1} a_{i} b_{i} r_{i}^{T}=\frac{1}{m_{k+1}} \sum_{i=1}^{k} a_{i} b_{i} r_{i}^{T}+\frac{1}{m_{k+1}} a_{k+1} b_{k+1} r_{k+1}^{T}
\end{aligned}
$$




$$
\begin{gathered}
S_{k+1} \triangleq B_{k+1}+B_{k+1}^{T}=B_{k}+B_{k}^{T}+\delta B_{k+1}+\delta B_{k+1}^{T} \\
z_{k+1} \triangleq \frac{1}{m_{k+1}} \sum_{i=1}^{k} a_{i}\left(b_{i} \times r_{i}\right)=\frac{1}{m_{k+1}} \sum_{i=1}^{k} a_{i}\left(b_{i} \times r_{i}\right)+\frac{1}{m_{k+1}} a_{k+1}\left(b_{k+1} \times r_{k+1}\right)
\end{gathered}
$$

Using the definitions in (11) and (12), (14.b) to (14.e) can be written as follows

$$
\begin{aligned}
& \sigma_{k+1}=\frac{m_{k}}{m_{k+1}} \sigma_{k}+\frac{1}{m_{k+1}} \delta \sigma_{k+1} \\
& S_{k+1}=\frac{m_{k}}{m_{k+1}} S_{k}+\frac{1}{m_{k+1}} \delta S_{k+1} \\
& z_{k+1}=\frac{m_{k}}{m_{k+1}} z_{k}+\frac{1}{m_{k+1}} \delta z_{k+1}
\end{aligned}
$$

When $\sigma_{k+1}, S_{k+1}$, and $z_{k+1}$ defined in (15) are used to form $K_{k+1}$, using the format of (6), (13) results. This ends the proof.

We have assumed here that we add only one new measurement to the $k$ measurements that were already processed. This can be extended to the case where two or more measurements are added as a group of measurements. Suppose that $\mathrm{K}$ was computed $\mathrm{n}$ times where at each time, one or more measurements were use to compute (initially) or to update $K$. Let this $K$ be denoted by $K_{n} n=1,2, \ldots$, where $K_{1}$ is computed using (5) and (6), and where the index $k$ is the number of measurements used to compute $K_{1}$. Suppose that $\mathrm{j}$ new pairs of vectors are measured and we want to use them in the updating of $\mathrm{K}$. We can, of course, update $K_{n} j$ times, using the algorithm presented in Proposition $I$, and obtain the updated $K$, or we can lump the new $j$ measurements together, and update $K$ only once. The latter is performed according to the algorithm listed in the following proposition.

Proposition 2: Let

$$
\delta m_{n+1}=\sum_{i=k+1}^{k+j} a_{i}
$$

where $k$ is the number of, already processed, pairs of vector measurements,

$$
\begin{gathered}
\delta \sigma_{n+1}=\sum_{i=k+1}^{k+j} a_{i} b_{i}^{T} r_{i} \\
\delta B_{n+1}=\sum_{i=k+1}^{k+j} a_{i} b_{i} r_{i}^{T} \\
\delta S_{n+1}=\delta B_{n+1}+\delta B_{n+1}^{T} \\
\delta z_{n+1}=\sum_{i=k+1}^{k+j} a_{i}\left(b_{i} \times r_{i}\right)
\end{gathered}
$$




$$
\delta K_{n+1}=\left[\begin{array}{l|l}
\delta S_{n+1}-\delta \sigma_{n+1} I & \delta z_{n+1} \\
\delta z_{n+1}^{T} & \delta \sigma_{n+1}
\end{array}\right]
$$

then

$$
m_{n+1}=m_{n}+\delta m_{n+1}
$$

and

$$
K_{n+1}=\frac{m_{n}}{m_{n+1}} K_{n}+\frac{1}{m_{n+1}} \delta K_{n+1}
$$

This proposition can be easily proven along the lines of the proof of the first proposition. The case described in the first proposition is a special case of the latter. we chose to split the introduction of the updating of $\mathrm{K}$ into two cases merely for methodical reasons.

\section{THE RECURSIVE TIME-VARYING ALGORITHM}

The updating algorithm of the static case can now be extended to the case where the body rotates between measurements. In the ensuing development we distinguish between two cases; namely, the error-free propagation case, and the propagation which is based on angular rate measurement, and as such, is contaminated by rate-measurement errors.

\section{III.1 Error-Free Propagation}

Assume that at time $\mathfrak{t}, \mathrm{k}$ pairs were processed, then the body rotated to a new orientation and there, at time $t_{n+1}$, j new vector measurements were performed. We wish to find the least squares fit of the quaternion to the first $k$ measurements, at this new time point, and then do the same when the new $j$ measurements are considered too. So first we are interested in finding $q_{n+1 / n}^{*}$ which is the quaternion that expresses best the attitude at time $t_{n+1}$, based on the first $k$ measurements which were performed previously, at time $t_{n}$. Let us re-write the cost function of (4) for $q$ at time $t_{n}$ based on the first $k$ measurements which, as mentioned, were performed at time $t_{n}$

$$
g\left(q_{n / n}\right)=q_{n / n}^{T} K_{n / n} q_{n / n}
$$

It is well known ${ }^{9}$ that during the rotation, $q$ changes according to the differential equation

$$
\dot{q}=\frac{1}{2} \Omega q
$$

where $\Omega$ is a $4 \times 4$ skew symmetric matrix whose elements are the body components of the vector of the angular velocity of the body with respect to the reference frame. The solution of (19) yields

$$
q\left(t_{n+1}\right)=\Phi\left(t_{n+1}, t_{n}\right) q\left(t_{n}\right)
$$

Ideally, when $\Omega$ is known perfectly, the matrix $\Phi\left(t_{n+1}, t_{n}\right)$, known as Transition Matrix, transforms the quaternion which represent the attitude at time $t_{n}$, to that which represents attitude at time $t_{n+1}$. For simplicity of notations, we denote it, simply, by $\Phi$. The quaternion which we wish to transform from time $t_{n}$ to time $t_{n+1}$ is $q_{n / n}$, thus we set $q\left(t_{n}\right)=q_{n / n}$. Finally, we denote the quaternion, to which $q_{n / n}$ is transformed, by $q_{n+1 / n^{\prime}}$ thus we set $q\left(t_{n+1}\right)=q_{n+1 / n}$. Consequently (18) becomes

$$
q_{n+1 / n}=\Phi q_{n / n}
$$


Since $\Omega$ is skew symmetric, $\Phi$ is orthogonal, thus we can write

$$
q_{n / n}=\Phi^{-1} q_{n+1 / n}=\Phi^{T} q_{n+1 / n}
$$

Substitution of $q_{n / n}$ of (22) into (18) yields

$$
g\left(q_{n / n}\right)=g^{\prime}\left(q_{n+1 / n}\right)=q_{n+1 / n}^{T} \Phi K_{n / n} \Phi^{T} q_{n+1 / n}
$$

We realize that the problem of finding $q_{n / n}$ that maximizes $g$ has been transformed into the problem of finding $\mathbf{q}_{n+1 / n}$ which maximizes $g^{\prime}$. Let

$$
K_{n+1 / n}=\Phi K_{n / n} \Phi^{T}
$$

then (23) becomes

$$
g^{\prime}\left(q_{n+1 / n}\right)=q_{n+1 / n}^{T} K_{n+1 / n} q_{n+1 / n}
$$

One may ask oneself whether the problem of finding $q_{n+1 / n^{\prime}}$, which maximizes $g^{\prime}$, is still related to Wahba's problem; that is, will the maximization of $g^{\prime}$ yield a quatemion which is a least squares fit to the $k$ vector measurements. The answer is, of course, positive, since the maximizing $q_{n+1 / n}$ is directly related, through (22), to $q_{n / n}$ which maximizes (18), and the latter is the solution of Wahba's problem, given the $k$ measurements. It can be shown (see the Appendix) that, like before, $q^{*}{ }_{n+1 / n}$, which is the $q_{n+1 / n}$ that maximizes $g^{\prime}$, given in (25), satisfies the equation

$$
K_{n+1 / n} q_{n+1 / 1}^{*}=\lambda_{n+1 / n} q_{n+1 / n}^{*}
$$

and that $q_{n+1 / n}^{*}$ is the eigenvalue of $K_{n+1 / n}$ which corresponds to the largest eigenvalue of $K_{n+1 / n}$. It is interesting to note that this solution to the constrained optimization problem is not specific to attitude determination. It stems from the fact that the cost function is defined as a quadratic form of a square matrix and that $q$ is required to be of unity length. (See the Appendix). Also note that although we assume error-free propagation, the measured vectors contain measurement errors. Finally, note that $K_{n+1 / n}$, being a result of a similarity transformation on $K_{n / n}$, has the eigenvalues of the latter even though its eigenvectors are different.

Now that we have established the fact that $K_{n+1 / n}$ is the proper $K$ matrix for finding the least squares fit of the quaternion at time $t_{n+1}$ based on all past $k$ measurements, we can include $j$ more measurements performed at $t_{n+1}$. For this we use (17.b) of Proposition II. Consequently from (24) and (17.b) we obtain

$$
\begin{gathered}
K_{n+1 / n}=\Phi K_{n / n} \Phi^{T} \\
K_{n+1 / n+1}=\frac{m_{n}}{m_{n+1}} K_{n+1 / n}+\frac{1}{m_{n+1}} \delta K_{n+1}
\end{gathered}
$$

We demonstrate the algorithm by way of the following example. 


\section{Example:}

Data base:

Given are 4 error free vectors in the reference coordinate frame:

$$
\mathbf{r} 1=\left[\begin{array}{l}
0.267 \\
0.535 \\
0.802
\end{array}\right] \quad \mathbf{r} 2=\left[\begin{array}{c}
-0.667 \\
-0.667 \\
-0.333
\end{array}\right] \quad \mathbf{r} 3=\left[\begin{array}{c}
0.267 \\
-0.802 \\
0.535
\end{array}\right] \quad \mathbf{r} 4=\left[\begin{array}{c}
-0.447 \\
0.894 \\
0.000
\end{array}\right]
$$

and a rotation from the reference to body axes described by the following Euler vector:

$$
\phi^{T}=[0.9,0.2,0.8]
$$

The corresponding quaternion is:

$$
q(1)^{T}=[0.423,0.094,0.376,0.819]
$$

The four $r$ vectors are transformed to the body frame and noise is added to the transformed vectors. The noise elements added to each component of the transformed vectors is drawn from a random number generator. The standard deviation of the noises are:

$$
\sigma 1=0.01 \quad \sigma 2=0.05 \quad \sigma 3=0.03 \quad \sigma 4=0.02
$$

The noise element added to each component of $r_{i}$ is drawn from the random number generator whose standard deviation is $\sigma_{i}, i=1,2,3,4$. The vectors are then normalized. The resulting simulated measured vectors in body frame are then:

$$
\mathbf{b} 1=\left[\begin{array}{l}
0.688 \\
0.662 \\
0.297
\end{array}\right] \quad \mathbf{b} 2=\left[\begin{array}{c}
-0.985 \\
-0.120 \\
-0.123
\end{array}\right] \quad \mathbf{b} 3=\left[\begin{array}{c}
-0.280 \\
-0.030 \\
0.959
\end{array}\right] \quad \mathbf{b} 4=\left[\begin{array}{c}
0.303 \\
0.575 \\
-0.760
\end{array}\right]
$$

and the weights are chosen to be:

$$
a_{i}=\sigma_{i}^{-2} \quad i=1,2,3,4
$$

Application of QUEST to the first two pairs:

Using, initially, at time $t_{1}$, the first two pairs of vectors, $r 1$ and $b 1$, and $r 2$ and $b 2$, we obtain $K_{1 / 1}$. Its largest eigenvalue and the corresponding eigenvector, which, according to our notations, is $q_{1 / 1}$ ' are:

$$
\lambda_{1 / 1}=1.0003551 \quad q_{1 / 1}^{\mathrm{T}}=[0.427,0.105,0.383,0.813]
$$

The corresponding transformation matrix, $A_{1 / 1}$, the correct matrix, $A(1)$, and the difference (error) matrix, are: 


$$
\begin{gathered}
A_{1 / 1}=\left[\begin{array}{ccc}
0.685 & 0.712 & 0.156 \\
-0.532 & 0.343 & 0.774 \\
0.497 & -0.613 & 0.614
\end{array}\right] \quad A(1)=\left[\begin{array}{ccc}
0.700 & 0.695 & 0.164 \\
-0.536 & 0.361 & 0.763 \\
0.471 & -0.622 & 0.625
\end{array}\right] \\
A_{1 / 1}-A(1)=\left[\begin{array}{ccc}
-0.015 & 0.017 & -0.007 \\
0.004 & -0.018 & 0.011 \\
0.026 & 0.009 & -0.012
\end{array}\right]
\end{gathered}
$$

The Euclidean norm of the error matrix is:

$$
\left|A_{1 / 1}-A(1)\right|=0.044
$$

This error stems, of course, from the measurement error contained in the $\mathbf{b}$ vectors.

Rotation of the body coordinate system:

We assume that after processing the first two pairs, which yielded $A_{1 / 1}$, the body rotates for 1 sec at the following angular rate:

$$
\omega^{\mathrm{T}}=[0.1,0.2,-0.3] \mathrm{rad} / \mathrm{sec}
$$

The matrix $\Phi$ which propagates the quatemion of this rotation (see (21)), and, $\Delta \mathrm{A}$, the attitude matrix which expresses the change in the body coordinates are:

$$
\Phi=\left[\begin{array}{cccc}
0.983 & -0.149 & -0.099 & 0.050 \\
0.149 & 0.983 & 0.050 & 0.099 \\
0.099 & -0.050 & 0.983 & -0.149 \\
-0.050 & -0.099 & 0.149 & 0.983
\end{array}\right] \quad \Delta \mathrm{A}=\left[\begin{array}{ccc}
0.936 & -0.283 & -0.210 \\
0.303 & 0.951 & 0.068 \\
0.181 & -0.127 & 0.975
\end{array}\right]
$$

Measurement update of $\mathrm{K}$ :

We use $\Delta A$ to transform $b_{3}$ and $b_{4}$ to the new time point, $t_{2}$. Using these $b$ 's as the simulated measurements at $t_{2}$, we compute $\delta K_{2}$ according to (16), and update $K$, using (27), as follows:

$$
\begin{gathered}
\mathrm{K}_{2 / 1}=\Phi \mathrm{K}_{1 / 1} \Phi^{\mathrm{T}} \\
\mathrm{K}_{2 / 2}=\frac{\mathrm{m}_{1}}{\mathrm{~m}_{2}} \mathrm{~K}_{2 / 1}+\frac{1}{\mathrm{~m}_{2}} \delta \mathrm{K}_{2}
\end{gathered}
$$

The largest eigenvalue and the corresponding eigenvector (which is $q_{2 / 2}$ ) of $K_{2 / 2}$ are:

$$
\lambda_{2 / 2}=1.0001957 \quad q_{2 / 2}^{\mathrm{T}}=[0.402,0.253,0.282,0.834]
$$


The corresponding attitude matrix is:

$$
A_{2 / 2}=\left[\begin{array}{ccc}
0.713 & 0.673 & -0.195 \\
-0.267 & 0.518 & 0.813 \\
0.648 & -0.528 & 0.549
\end{array}\right]
$$

Check:

We wish, now, to check this result. This is done as follows. We use $\Delta A$ to transform $b_{1}$ and $b_{2}$ to the new time point, $t_{2}$. (Recall that $b_{3}$ and $b_{4}$ were already transformed in order to compute $\delta K_{2}$ ). Now we apply the QUEST algorithm to all four pairs of $\mathbf{r}$ and $\mathbf{b}$. The resulting quaternion should be equal to the quatemion updated by the REQUEST algorithm. Indeed the two quatemions agree to at least $10^{-12}$.

Remark:

When we compare, $A(2)=\Delta A \cdot A(1)$, which is the correct matrix which transforms from the reference to body axes at $t_{2}$, to the attitude matrix $A_{2 / 2}$, obtained by REQUEST (and, as we just checked, by QUEST as well), we obtain:

$$
A_{2 / 2}-A(2)=\left[\begin{array}{ccc}
0.005 & -0.006 & 0.000 \\
-0.001 & 0.007 & -0.005 \\
-0.006 & -0.001 & 0.007
\end{array}\right]
$$

The Euclidean norm of this error matrix is:

$$
\left|A_{2 / 2}-A(2)\right|=0.015
$$

This error stems from the measurement noise in the $\mathbf{b}$ vectors and not from the algorithm. We note that the latter error is smaller than $\left|A_{1 / 1}-A(1)\right|$ shown in (28). This is expected, since $A_{2 / 2}$ is computed using four pairs of vectors whereas $A_{1 / 1}$ is computed using only two.

\section{III.2 Noisy Propagation}

In the preceding developments we considered the presence of noise only in the measurements and assumed that the angular rate vector, $\omega$, was known to us perfectly. We wish to consider now errors also in our knowledge of $\omega$. Let us denote the measured, or computed, $\omega$ by $\omega_{\mathrm{m}}$. We also assume that the error is additive, thus we can write

$$
\omega_{\mathrm{m}}=\omega+\varepsilon
$$

where $\varepsilon$ is the error component in the measured angular rate vector. We distinguish between two cases; namely, short time application, and long time application of REQUEST. The two are treated next.

\section{III.2.1 Short Mission Duration}

Since a typical update rate is once per second, typical gyro noise does not cause a considerable attitude error during such a short period. In fact, even with an update rate of once per 10 seconds, the attitude error amounts to a very small attitude error. To illustrate this point, we turn to the example. Suppose that we use a triad of single axis gyros, each having a constant drift rate of $1^{\circ} \mathrm{h}$, 
which is about 100 times larger than that of inertial grade gyros. We use the first three measurements to compute $K_{1 / 1}$ and from it, $A_{1 / 1}$. We then propagate $K_{1 / 1}$ using $\Phi_{m}$, the gyro-error ridden transition matrix, and obtain $K_{2 / 1, m}$ and then compute the corresponding attitude matrix, $A_{2 / 1, m}$. In parallel we do the same using $\Phi$, the correct transition matrix, and obtain $A_{2 / 1}$, the corresponding attitude matrix. Doing so, we discover that the largest difference between the magnitude of the elements of the two attitude matrices, $A_{2 / 1, m}$ and $A_{2 / 1}$, is less than $5.23 \cdot 1 \mathrm{C}^{-6}$. Next, following the REQUEST algorithm, we use the fourth measurement at time $t_{2}$ to compute $\delta K_{2}$, update both, $K_{2 / 1, m}$ and $K_{2 / 1}$, and compute the corresponding attitude matrix for the correct and erroneous propagations. The largest error between the elements of the, updated, two attitude matrices is less than $2.55 \cdot 10^{-6}$. We see two interesting facts. First, indeed, the gyro error has little effect on the propagated $K$ and, consequently, on the, propagated and the updated, attitude matrices. Second, the incorporation of a new measurement reduces the little error, caused by gyro drift, even further. As a consequence of this discussion, we conclude that for a short mission duration the build up of attitude errors as a result of gyro drift is negligible and the algorithm given in (27) is adequate.

\section{III.2.2 Long Mission Duration}

Space missions where QUEST is traditionally being used, are of long duration, therefore the initial measurements are propagated through the repeated use of (27.a) to the current time. This in turn reduces the accuracy of those measurements, and as time goes by they may corrupt the attitude rather than improve it. Consequently, we wish to gradually reduce the influence of old measurements, and eventually eliminate them altogether. This is usually done using the Fading Memory concept. Accordingly, instead of using (27.b) for updating $\mathrm{K}$, we may want to use the following algorithm

$$
K_{n+1 / n+1}=\rho_{n} \frac{m_{n}}{m_{n+1}} K_{n+1 / n}+\frac{1}{m_{n+1}} \delta K_{n+1}
$$

where $0<\rho_{n} \leq 1$. Note that $\rho_{n}$ has to be larger than 0 for (28.a) to yield a meaningful $K$ when only one measurement is performed at $t_{n+1}$. Also note that when no process noise is present, we set $\rho_{n}=1$ which keeps the same relative weighting of past and present measurements as in (27.b). The value of $\rho_{n}$ can be determined experimentally where a larger propagation noise is compensated by a smaller $\rho_{n}$ value. Note that $p_{n}$ can vary from step to step allowing the consideration of changing gyro noise. It should be noted that the introduction of $m_{n}$ in the REQUEST algorithm stems from our wish to maintain $\lambda_{\max } \cong 1$. This is important if we use the classical QUEST method for solving for $\lambda_{\max }$ [see ref. 7]. (If we use a given eigenvalue-eigenvector solver routine, this is irrelevant). When (28.a) is used and we are still interested in having $\lambda_{\max }$ close to 1 , we have to replace (28.a) by

$$
K_{n+1 / n+1}=\frac{\rho_{n} m_{n}}{\rho_{n} m_{n}+\delta m_{n+1}} K_{n+1 / n}+\frac{1}{\rho_{n} m_{n}+\delta m_{n+1}} \delta K_{n+1}
$$

Note that, as before, this $\mathrm{K}$ update algorithm still assures proper weighing of the measurements; that is, the measurement noise is properly considered.

\section{ALGORITHM SUMMARY}

The REQUEST algorithm is summarized as follows.

1. Use the $k$ measurements performed at the starting point, $t_{1}$, tocompute $K_{1 / 1}$. First compute: 


$$
\begin{gathered}
m_{k}=\sum_{i=1}^{k} a_{i} \\
\sigma=\frac{1}{m_{k}} \sum_{i=1}^{k} a_{i} b_{i}^{T} r_{i} \\
B=\frac{1}{m_{k}} \sum_{i=1}^{k} a_{i} b_{i} r_{i}^{T} \\
S=B+B^{T} \\
z=\frac{1}{m_{k}} \sum_{i=1}^{k} a_{i}\left(b_{i} \times r_{i}\right)
\end{gathered}
$$

Then compute:

$$
\mathrm{K}_{1 / 1}=\left[\begin{array}{c|c}
S-\sigma \mathrm{I} & \mathrm{z} \\
\hline \mathrm{z} & \sigma
\end{array}\right]
$$

2. Form the angular rate matrix:

$$
\frac{1}{2} \Omega=\frac{1}{2}\left[\begin{array}{cccc}
0 & \omega_{z} & -\omega_{y} & \omega_{x} \\
-\omega_{z} & 0 & \omega_{x} & \omega_{y} \\
\omega_{y} & -\omega_{x} & 0 & \omega_{z} \\
-\omega_{x} & -\omega_{y} & -\omega_{z} & 0
\end{array}\right]
$$

where, $\omega_{i}, i=1,2,3$ are the components of the body axes, angular rate vector.

3. Compute $\Phi$, the transition matrix from time $t_{1}$ to time $t_{2}$, corresponding to this, generally time-varying, angular rate matrix. (Algorithms for computing $\Phi$ can be found in standard Control Theory or State Estimation texts. See e.g. Gelb ${ }^{11}$.)

4. Propagate $K_{1 / 1}$ according to:

$$
K_{2 / 1}=\Phi K_{1 / 1} \Phi^{T}
$$

5. Compute $\delta \mathrm{K}_{2}$ as follows:

$$
\delta m_{2}=\sum_{i=\frac{k}{k+1}}^{k+j} a_{i}
$$

(where $k$ is the number of, already processed, pairs of vector measurements, and $j$ is the number of new measurement pairs performed at time $t_{2}$ ).

$$
\begin{aligned}
& \delta \sigma_{2}=\sum_{i=k+1}^{k+j} a_{i} b_{i}^{T} r_{i} \\
& \delta B_{2}=\sum_{i=k+1}^{k+j} a_{i} b_{i} r_{i}^{T} \\
& \delta S_{2}=\delta B_{2}+\delta B_{2}^{T} \\
& \delta z_{2}=\sum_{i=k+1}^{k+j} a_{i}\left(b_{i} \times r_{i}\right)
\end{aligned}
$$


then set $\rho_{n}$ in the range

$$
\delta K_{2}=\left[\begin{array}{c|c}
\delta S_{2}-\delta \sigma_{2} I & \delta z_{2} \\
\hline \delta z_{2}^{T} & \delta \sigma_{2}
\end{array}\right]
$$

and compute

$$
0<\rho_{n} \leq 1
$$

$$
K_{2 / 2}=\frac{\rho_{1} m_{1}}{\rho_{1} m_{1}+\delta m_{2}} K_{2 / 1}+\frac{1}{\rho_{1} m_{1}+\delta m_{2}} \delta K_{2} .
$$

In preparation for the next time update, compute

$$
m_{2}=m_{1}+\delta m_{2}
$$

6. Only if there is an interest in extracting the attitude from $\mathrm{K}_{22}$, compute the attitude at this time point $\left(t_{2}\right)$, otherwise go to step 7. The extraction of attitude from $K_{2 / 2}$ can be done using the algorithm given in QUEST, or any standard software package that can compute eigenvalues and eigenvectors of a symmetric matrix (e.g. Matlab ${ }^{\mathrm{TM}}$ or Mathcad ${ }^{\mathrm{TM}}$ ). If the latter approach is chosen, then, first, select the largest eigenvalue of $K_{2 / 2}$, and, then, compute the corresponding eigenvector.

7. Go to step 2 and increase the appropriate indices by 1 , or stop if so desired.

\section{CONCLUSIONS AND RECOMMENDATIONS}

In this work we presented a recursive algorithm for attitude determination, from vector observations, that was derived from QUEST. The new recursive algorithm, which we call REQUEST, is based on the propagation and update of the $\mathrm{K}$ matrix, one of whose eigenvectors is the sought attitude quaternion. Using REQUEST, we do not lose information gathered by measurements performed at previous time points, and since we use prior information, even one measurement at a particular time point, to which $K$ is propagated, is sufficient for updating the attitude. We showed how to apply the algorithm to cases where more than one measurement is taken at the new time point. We demonstrated that under normal conditions, and for short mission durations, there is no need to treat propagation noise (also known as process noise). For long mission durations we do have to consider the process noise. This is done using the Fading-Memory notion whereby the weight of the contribution of old measurements to $K$ is reduced with time. We presented an example to illustrate the algorithm.

As mentioned, the new algorithm shows how the propagate and update $K$, but once $K$ is computed, its largest eigenvalue and the corresponding eigenvector, which is the sought quaternion, are found using the method of QUEST. If, however, a standard eigenvalue-eigenvector solver algorithm, is used, then the eigenvalue and eigenvector can be found directly without solving for Rodrigues parameters, and without the need to be concerned about matrix singularity problems (see (9)).

As a follow up to this work, it is recommended that REQUEST be tested using real spacecraft data, and be tested against other recursive algorithms, such as the extended Kalman filter.

\section{Acknowledgement}

We wish to thank P. Landis Markley for his helpful comments, particularly on the damping of past measurements in long duration missions. 


\section{Appendix}

In this appendix we prove that a cost function formulated as a quadratic form of a real symmetric marrix, with a unity constraint on the vector part of this form, has the following two qualities:

I. Its maximum is equal to the value of the matrix largest eigenvalue.

II. The vector which maximizes the cost function is the matrix eigenvector which corresponds to this eigenvalue

We present the proof in a form of a question and an answer as follows.

Problem: Given

$$
\mu=\mathrm{x}^{\mathrm{T}} \mathrm{M} \mathbf{x} \quad \text { (A.1) where } \quad|\mathbf{x}|=1
$$

and $\mathrm{M}$ is an $\mathrm{n} \times \mathrm{n}$ symmetric matrix, find $\mathrm{x}$ which maximizes $\mu$.

Solution: We use the method of Lagrange multipliers to incorporate the constraint of (A.2) in the cost function expressed in (A.1). Accordingly, we wish to maximize $\varphi(x)$ given by

$$
\varphi(\mathbf{x})=\mathbf{x}^{\mathrm{T}} \mathbf{M} \mathbf{x}+\lambda\left(1-\mathbf{x}^{\mathrm{T}} \mathbf{x}\right)
$$

We denote the maximizing $x$ by $x^{*}$, then we can express $x$ as follows

$$
\mathbf{x}=\mathbf{x}^{*}+\boldsymbol{\varepsilon h}
$$

where $\varepsilon$ is a scalar. Substitution of the latter into (A.3) yields

$$
\phi(\varepsilon)=\left(x^{*}+\varepsilon h\right)^{T} M\left(x^{*}+\varepsilon h\right)+\lambda\left[1-\left(x^{*}+\varepsilon h\right)^{T}\left(x^{*}+\varepsilon h\right)\right]
$$

An extremal point of $\phi(\varepsilon)$ satisfies the following

$$
\left.\frac{\mathrm{d} \phi(\varepsilon)}{\mathrm{d} \varepsilon}\right|_{\varepsilon=0}=0 \quad \text { for all } h
$$

Now it can be easily verified that since $M$ is symmetric,

$$
\left.\frac{d \phi(\varepsilon)}{d \varepsilon}\right|_{\varepsilon=0}=2 h^{T}\left(M x^{*}-\lambda x^{*}\right)
$$

Application of the condition for a stationary point of (A.6) to (A.7), yields

$$
h^{T}\left(M x^{*}-\lambda x^{*}\right)=0 \quad \text { for all } h
$$

The latter condition can be met if and only if

$$
\mathbf{M x}^{*}=\lambda \mathbf{x}^{*}
$$

Substitution of $\mathrm{Mx}^{*}$ given by the last equation into (A.1) yields

$$
\mu_{\max }=\lambda \mathbf{x}^{*} \mathbf{x}^{*}
$$

and since $x^{*}$ is of unit length, $x^{*} x^{*}=1$, therefore (A.10) becomes

$$
\mu_{\max }=\lambda
$$

and $\mu_{\max }$ takes its maximal value when $\lambda$ is $\lambda_{\max }$, which is the largest eigenvalue of

$M$. (Note that since $M$ is symmetric, its eigenvalues are always real). Then

$$
\mu_{\max }=\lambda_{\max }
$$

and $x^{*}$ is the eigenvector of $M$ which corresponds to $\lambda_{\max }$. 


\section{References}

1. Wahba, G., "A Least Squares Estimate of Spacecraft Attitude," SIAM Review, Vol. 7, No. 3, July 1965, p.409.

2. Davenport, P., Private Communication, 1968.

3. Keat, J., Analysis of Least-Squares Attitude Determination Routine DOAOP, Computer Sciences corporation, CSC/TM-77/6034, Feb. 1977.

4. Rodrigues, M.O., "Des Lois Géométriques Qui Régissent les Déplacement d'un Système Solide dans L'espace, et de la Variation des Coordonnées Provenant de ces Déplacements Considérés Independamment des Causes qui Peuvent les Produire," Journal de Mathematiques Pures et Appliquees. (Liouville), Vol. 5, 1840, pp. 380-440.

5. Wertz, J.R., (ed), Spacecraft Attitude Determination and Control, D. Reidel Publishing Co., Dordrecht, Holland, 1984, p. 512, 513.

6. Shuster, M.D., Algorithms for Determining Optimal Attitude solutions, Computer Sciences Corporation, CSC/TM-78/6056, April 1978.

7. Shuster, M.D., and Oh, S.D., "Three-Axis Attitude Determination from Vector Observations," Journal of Guidance and Control, Vol. 4, No. 1, Jan.-Feb, 1981, pp. 70-77.

8. Shuster, M.D., "A Simple Kalman Filter and Smoother for Spacecraft Attitude," Journal of the Astronautical Sciences, Vol. 37, No. 1, 1989, pp. 89-106.

9. Wertz, J.R., (ed), Spacecraft Attitude Determination and Control, D. Reidel Publishing Co., Dordrecht, Holland, 1984, p. 512.

10. Gelb, A., (ed), Applied Optimal Estimation, The M.I.T. Press, Cambridge, MA, 1988, pp. 285-287.

11. Ibid., 57-63, 296-298. 
